\title{
MECHANICS OF MATERIALS: TOP-DOWN APPROACHES TO FRACTURE*
}

\author{
J. W. HUTCHINSON ${ }^{\dagger} \uparrow$ and A. G. EVANS ${ }^{2}$ \\ ${ }^{1}$ Division of Engineering and Applied Sciences, Harvard University, Cambridge, MA 02138, USA and \\ ${ }^{2}$ Materials Institute, Princeton University, Princeton, NJ 08540, USA
}

(Received 1 June 1999; accepted 15 July 1999)

\begin{abstract}
The utility and robustness of the mechanics of materials is illustrated through a review of several recent applications to fracture phenomena, including adhesive failures, the role of plasticity in enhancing toughness in films and multilayers, and crack growth resistance in ductile structural alloys. The commonalty among the approaches rests in a reliance on experiments to provide calibration of the failure process at the smallest scale. (C) 2000 Acta Metallurgica Inc. Published by Elsevier Science Ltd. All rights reserved.
\end{abstract}

Keywords: Mechanics of materials; Mechanical properties; Fracture and fracture toughness; Plastic properties

\section{INTRODUCTION}

Starting with earlier applications in elasticity and plasticity, the mechanics of materials has evolved in recent decades into topics related to the failure of structural metals, ceramics and polymers, as well as composites, coatings and multilayers. Methods have been developed for assessing the performance of advanced materials in new applications and designs, through models of deformation, damage and fracture. Most methods have some element of phenomenology, especially when small-scale mechanisms influence macroscopic behavior. Yet, mechanismbased concepts provide key insights. The canonical example is provided by the fracture mechanics of structural materials. Fracture mechanics has established a framework for: (i) experimentally measuring the fracture resistance of a material under monotonic and cyclic loadings; and (ii) assessing structural integrity using these data. The extraordinary success of fracture mechanics lies in its ability to combine a theoretical framework with experimentally measured critical quantities. The mechanics is used to link the macroscopic geometry and loads to microscopic fracture processes, which are then characterized by experiment, not theory. Despite significant progress in the theoretical under-

$\dagger$ To whom all correspondence should be addressed.

The Millennium Special Issue - A Selection of Major Topics in Materials Science and Engineering: Current status and future directions, edited by S. Suresh. standing of the influence of microstructure on fracture, the development of predictive models continues to provide one of the major mechanics and physics challenges. It remains true that material fracture properties are experimentally measured quantities in nearly all present day applications of fracture mechanics, whether toughness, fatigue or stress corrosion growth rates.

The theme of this paper is the exploration of the considerable additional scope within the mechanics of materials for further significant advances in "top-down" approaches, which couple continuum mechanics descriptions to phenomenology and experimental calibration at the smallest scales. In most structural material systems, given the complexity of the microscopic processes, "bottom-up" approaches which use fundamental mechanics and physics to link the atomic scale to the macroscopic aspects of deformation and fracture are unlikely to be developed with adequate accuracy in the near future.

Four interrelated topics will be used to illustrate this point of view, each selected within the scope of problems to be found in crack growth and adhesion (Fig. 1). The general plan is to augment the conventional approach to crack growth, which is based on a single parameter (namely, the local energy release rate, $\left.G_{\text {tip }}\right)$, with a richer model capable of incorporating microscopic aspects of the rupture process itself. The underlying concept is to divide the process into two separate domains that can be analyzed independently and then linked together to express 




Fig. 1. Schematic of fracture models which employ an embedded fracture process zone (EPZ) within continuum descriptions of adjoining solids. Some models for mode I separation specify a traction-separation, $\sigma(\delta)$, on the crack plane. A more elaborate model representing the ductile fracture mechanism of void nucleation, growth and coalescence uses calibrated elements which simulate the ductile fracture mechanism at various states of stress triaxiality.

the overall behavior. One domain represents the zone near the crack front that may experience very large strains as the fracture process evolves. This zone incorporates a model of the rupture process, referred to as an embedded process zone (EPZ). The other domain is the physically larger plastic zone and outer elastic region that can be analyzed using either continuum models of elastic/plastic behavior, or variants that incorporate a plasticity length scale. The link between the two zones is provided by tractions, $\sigma$, and displacements, $\delta$, on the boundary between the zones, resulting in the wellknown leveraging effect of the EPZ response on the size of the inelastic zone and, accordingly, on the overall crack growth resistance. The details inherent in $\sigma(\delta)$ contain the information about the rupture mechanism, which replaces $G_{\text {tip }}$. Parameters characterizing $\sigma(\delta)$ will generally be determined by fitting model predictions to a selected set of experiments, thereby providing a calibration against the fracture process at the smallest relevant scale. Models for the EPZ have attained various levels of completeness. At the simplest level, the EPZ has been located on the fracture plane, subject to normal and shear tractions that express the rupture phenomenon. More complete models, such as that for the ductile fracture of structural alloys, incorporate the full effects of the multi-axial stress field in the EPZ. To explain this spectrum of models, the article is organized in four sections.

1. The EPZ model is used to extend fracture mechanics to adhesive interfaces between two elastic materials.

2. The linkage between the EPZ and plastic dissipation is explored.

3. EPZ models for fracture initiation and crack growth in ductile structural alloys are addressed subject to small- and large-scale yielding conditions.

4. Plasticity at the micrometer scale and its role in strength and toughness is examined.

\section{EPZ MODELS FOR ADHESIVE INTERFACES}

Important aspects of failure lie outside the scope of conventional elastic fracture mechanics, even 




Fig. 2. The four-point bending specimen used by Mohammed and Liechti [1] to study initiation of cracking along a brittle epoxy-aluminum. The role of non-cracklike stress concentrations on the load at fracture of the interface was determined. The distance between the loads was fixed at $254 \mathrm{~mm}$ and at $177.8 \mathrm{~mm}$ between the supporting rollers. The thickness of the $\mathrm{Al}$ and epoxy layers was fixed at $12.7 \mathrm{~mm}$.

under nominally elastic conditions. The situation is exemplified by fracture initiating from regions of stress concentration. A recent study on the fracture of brittle adhesive interfaces illustrates the EPZ approach [1]. The interface between the relatively brittle epoxy and the aluminum is the weak plane. The initial geometry does not have a crack at the interface, just a notch with an opening angle, $\alpha$ (Fig. 2). The elastic behavior in the vicinity of the notch is not governed by the inverse square root singularity characteristic of an interface crack, and accordingly, when $\alpha \neq 0$, the energy release rate is zero. Consequently, conventional elastic fracture mechanics cannot be used to predict the load at which the interface will fail.

Before elaborating on this problem, some of the details of the EPZ approach are summarized for the interface model illustrated in the lower left-hand corner of Fig. 1. The primary parameters characterizing the interface are the work of separation per unit area, $\Gamma_{0}$, and the peak stress, $\hat{\sigma}$. The shape of the separation relation is of secondary importance: once the shape is fixed, the critical separation, $\delta_{\mathrm{c}}$, is no longer an independent parameter. When separation takes place under both normal and tangential crack face displacements, a potential can be used to generate the relation between the traction components and the displacements such that the work of separation remains as $\Gamma_{0}$. Alternatively, a modedependent work of separation for the interface could be considered, but in this paper $\Gamma_{0}$ will be taken to be mode-independent.

Computation of the traction-separation behavior



Fig. 3. Comparison of measured loads at which crack growth on the interface initiated with predictions from the cohesive zone model [1]. Data were taken for $\alpha=0^{\circ}, 45^{\circ}$, $90^{\circ}$ and $135^{\circ}$. Only $\alpha$ was varied from specimen to specimen.

from first principles is beyond present capabilities. Mohammed and Liechti [1] determined the interface parameters from measurements made using a sharp crack test. No plastic deformation occurred in either the epoxy or the aluminum, apart from highly localized strains at the scale of the fracture process. The work of separation $\left(\Gamma_{0}=4.09 \mathrm{~J} / \mathrm{m}^{2}\right)$ was identified with the fracture toughness obtained with $\alpha=0$, whereupon, $\hat{\sigma}=3 \mathrm{MPa}$, gave the best fit to the opening displacement distribution at the verge of fracture. This stress is a small fraction of the tensile yield strength of the epoxy $\left(\sigma_{\mathrm{Y}}=20 \mathrm{MPa}\right)$, which accounts for the absence of bulk plasticity.

The success of the cohesive zone model in reproducing the experimentally measured loads at which the interface fails is seen in Fig. 3. While the coincidence of the simulation and test data for the sharp crack limit $(\alpha=0)$ necessarily follows from the calibration, the significant increase in failure load with increasing $\alpha$ is precisely captured by the model. Neither conventional fracture mechanics nor a critical stress approach could be used to predict this trend.

\section{EXTENSIONS TO ACCOUNT FOR EXTRINSIC PLASTIC DEFORMATION}

When the peak separation stress, $\hat{\sigma}$, is larger than two or three times the tensile yield strength of the softer of the two bonded materials, a significant plastic zone will be induced (Fig. 1) such that the total work of fracture will exceed $\Gamma_{0}$. To facilitate 




Fig. 4. The extrinsic plasticity contribution to plane strain steady-state toughness as predicted by the cohesive zone model for mode I crack growth along an interface separating identical solids with tensile yield stress $\sigma_{\mathrm{Y}}$ and strain hardening exponent $N$ [3]. The curves give the ratio of the steady-state macroscopic work of fracture to the intrinsic work of separation as a function of the ratio of peak separation stress to yield stress.

understanding of the effect of this zone, consider small-scale yielding (SSY) conditions wherein the height of the zone is small compared with the crack length, such that the energy release rate, $G$, still serves as the measure of the remote, or macroscopic, intensity. After the crack has propagated a distance comparable to the plastic zone size, steady state is approached with the crack advancing at a constant applied energy release rate with $G=\Gamma_{\text {ss }}$.

Application of the EPZ model has been made for strong interfaces in the sense that: $\hat{\sigma} / \sigma_{\mathrm{Y}}>2$ [2-5]. Plots of $\Gamma_{\mathrm{ss}} / \Gamma_{0}$ as a function of $\hat{\sigma} / \sigma_{\mathrm{Y}}$ for several values of strain hardening exponent, $N$, are given in Fig. 4 for plane strain, mode I growth with identical solids on either side of the interface. These results illustrate the large influence of extrinsic plasticity on the macroscopic toughness of strong interfaces. Mixed mode loading and asymmetry in yielding across the interface will affect the details, but the trends in Fig. 4 are representative. In particular, the exceptionally strong influence of the interface strength, $\hat{\sigma}$, is evident. With fixed $\hat{\sigma}$, the steady-state toughness $\Gamma_{\mathrm{ss}}$ scales linearly with $\Gamma_{0}$. Conversely, for fixed $\Gamma_{0}, \Gamma_{\mathrm{ss}}$ is an exceptionally strong nonlinear function of $\hat{\sigma}$. In this sense, changes in the interface strength have more effect on macroscopic toughness than changes in the intrinsic work of fracture.

In multilayer systems, an elastic layer (thickness $h_{0}$ ) may separate the interface crack from the ma-



a)

b)

Fig. 5. (a) Multilayers tested using edge-notched, four-point bend specimens by Dauskardt et al. [6] The interface that fractures lies between two sets of elastic layers: the $\mathrm{SiO}_{2}$ layer and the TiN/Ti/TiN layers. Plastic deformation occurs in the $\mathrm{Al}-\mathrm{Cu}$ layer. (b) Solid points indicate the measured interface toughness as a function of the thickness $h$ of the $\mathrm{Al}-\mathrm{Cu}$ layer. The curve is the theoretical calculation [7] based on the EPZ model in the SSV limit with the system parameters listed in the text. 




a)



Fig. 6. (a) Dependence of normalized steady-state toughness in mode I on normalized interface strength for a relatively thick $\mathrm{Al}-\mathrm{Cu}$ layer and six values of the normalized thickness of the intervening elastic layers. (b) Dependence of $\Gamma_{\mathrm{ss}} / \Gamma_{0}$ on mode mixity $\psi$ for a relatively thick $\mathrm{Al}-\mathrm{Cu}$ layer and three values of $h_{0} / R_{0}$ as computed from the SSV limit of the cohesive zone model [7].

terial layer susceptible to plastic deformation (thickness $h$ ). A specific example will be considered below. The intervening elastic layer introduces some new features, discussed next. The interface is governed by a traction-separation potential, again characterized by $\Gamma_{0}$ and $\hat{\sigma}$. If the plastic zone height is very small compared to the crack length and the total multilayer thickness, the applied loads can be specified in terms of $K_{\mathrm{I}}$ and $K_{\mathrm{II}}$ or, equivalently, by $G=\left(1-v^{2}\right)\left(K_{\mathrm{I}}^{2}+K_{\mathrm{II}}^{2}\right) / E$ and $\psi=\tan ^{-1}\left(K_{\mathrm{II}} / K_{\mathrm{I}}\right)$. If $\psi$ is prescribed, the history of $G$ vs crack advance, $\Delta a$, can be computed. A steady state is approached such that $G$ becomes independent of crack advance, and, in this limit, $G=\Gamma_{\text {ss }}$. A material-based length parameter that plays an important role is: $R_{0}=$ $\left[1 / 3 \pi\left(1-v^{2}\right)\right]\left[E \Gamma_{0} / \sigma_{\mathrm{Y}}^{2}\right]$. This reference length can be interpreted as a rough estimate of the height of a plastic zone which would arise in a thick metal layer directly in contact with the interface when the applied loading is $G \approx \Gamma_{0}$. It serves as the relevant length quantity against which both $h$ and $h_{0}$ should be compared.

Recent experiments on multilayers representative of those encountered in electronics on-chip provide an example (Dauskardt et al. [6]). The weakest interface in the multilayer (Fig. 5) is that between the $\mathrm{TiN}$ and the $\mathrm{SiO}_{2}$ layers. The three layers above this interface $(\mathrm{TiN} / \mathrm{Ti} / \mathrm{TiN})$ have a fixed combined thickness, $h_{0}=0.17 \mu \mathrm{m}$ and, because of their high hardness, deform elastically. Above these is a relatively soft layer of $\mathrm{Al}-\mathrm{Cu}$ having thickness, $h$, varying between 0.1 and $4 \mu \mathrm{m}$ in the seven configurations fabricated and tested. The multilayer is sandwiched between two relatively thick layers of $\mathrm{Si}$ to form a mixed mode flexure specimen that is then used to measure the interface toughness. The combined thickness of the multilayers is very small compared to both the interface crack length and the outer Si layers. The Young's moduli of the constituent materials differ by only a few percent, and for modeling purposes, are considered to be the same. The $\mathrm{Al}-\mathrm{Cu}$ layer is taken to be elastic-perfectly plastic with tensile yield strength $\sigma_{\mathrm{Y}}$ and a von Mises yield surface. The other layers are assumed to undergo only elastic deformations.

The calculated effect of the interface strength, $\hat{\sigma}$, on the steady-state toughening enhancement, $\Gamma_{\mathrm{ss}} / \Gamma_{0}$, in mode I is shown in Fig. 6(a) for several choices of $h_{0}$ and a relatively thick metal layer [7]. Weak interfaces $\left(\hat{\sigma} / \sigma_{Y}<2\right)$ give rise to very little toughness enhancement. The interface separates before significant plastic deformation occurs. The toughness enhancement becomes independent of the interface strength when $\hat{\sigma} / \sigma_{\mathrm{Y}}$ exceeds about four. This is the limit in which the behavior in the elastic region fully surrounding the crack tip is controlled by the local stress intensity factors and there is no difficulty attaining the separation stress. In this limit, the model only requires attainment of the local energetic condition, $G_{\text {tip }}=\Gamma_{0}$ [8]. This is the regime in which the SSV model of Suo, Shih and Varias [9] applies. (The elastic strip shielding the crack tip from the plastic deformation was introduced in the original SSV model to simulate a dislocation-free region near the tip). The effect of mixed mode loading on the steady-state toughness enhancement is shown in Fig. 6(b) for several values of $h_{0} / R_{0}$. These were obtained using the SSV limit of the cohesive zone model [7]. The asymmetry in the relation of $\Gamma_{\mathrm{ss}} / \Gamma_{0}$ to $\psi$ stems from the asymmetric location of the layer susceptible to plasticity. The mode I toughness is close to the lowest value, while the mode II component amplifies the toughness. The strong mixed mode toughness variation seen in Fig. 6(b) is due entirely to extrinsic plastic deformation.

The following values for the system parameters will be used in comparing the model simulations with the experimental results: $E=107 \mathrm{GPa}$, $v=0.25, \quad \sigma_{\mathrm{Y}}=140 \mathrm{MPa}, \quad h_{0}=0.17 \mu \mathrm{m} \quad$ and $\Gamma_{0}=1.5 \mathrm{~J} / \mathrm{m}^{2}$. For this set of values, $R_{0}=0.93 \mu \mathrm{m}$. The roles of the two remaining parameters, $h$ and $\hat{\sigma}$, will be explored. The specimen (Fig. 5) corresponds to a mixed mode loading with $\psi=40^{\circ}$. Simulations using the cohesive zone model in the 



Fig. 7. Peel test and results of EPZ model for sheet metal adhesive joint (Yang et al. [12]).

SSV limit have been made [7]. The model predictions are included along with the experimentally measured interface toughness as the solid curve in Fig. 5(b). The toughness enhancement with increasing thickness of the $\mathrm{Al}-\mathrm{Cu}$ is again entirely due to extrinsic plasticity since the interface is the same for all specimens. The model captures the trend of the data reasonably well and, in particular, brings out the relevance of the reference length $R_{0}$ to the thickness of the $\mathrm{Al}-\mathrm{Cu}$ layer and that of the elastic layers imposed between the layer and the interface. If $h / R_{0}>4$, the $\mathrm{Al}-\mathrm{Cu}$ layer can be regarded as thick in the sense that the plastic zone does not extend entirely across it and the macroscopic toughness becomes independent of $h$.

There are two major uncertainties in the comparison carried out in Fig. 5(b). Perhaps the most significant is the uncertainty in the yield strength $\sigma_{\mathrm{Y}}$ of the $\mathrm{Al}-\mathrm{Cu}$ which strongly affects the model predictions. Direct measurements of the yield stress on similar $\mathrm{Al}-\mathrm{Cu}$ films in the same range of thickness (by indentation and tensile testing) give $\sigma_{\mathrm{Y}}$ in the range from 100 to $200 \mathrm{MPa}$ [10]. Had the higher value been used in the simulation, the plasticity enhancement of the toughness would have been considerably less than that shown in Fig. 5(b), mainly due to a decrease in $R_{0}$ and the consequent increase in $h_{0} / R_{0}$ (cf. Fig. 6). There is also uncertainty in the intrinsic work of interface separation, $\Gamma_{0}$. This quantity has not been measured directly.
The choice $1.5 \mathrm{~J} / \mathrm{m}^{2}$ appears to be a reasonable extrapolation of the experimental data in Fig. 5(b), but by no means unambiguous, as already noted. At the present stage of development, without further refinement and verification, the model is still qualitative. Nevertheless, the ability to predict the major trends with respect to the coupled effects of mixed mode loading and extrinsic plasticity holds considerable promise for dealing with interface fracture in small-scale devices.

A successful application of the EPZ model to the failure analysis of adhesively bonded sheet metal has been initiated by Yang et al. [11, 12], motivated by efforts in the automotive industry to exploit adhesive joining technology. These authors have tested and analyzed tearing modes of adhesive joints under various loading modes. The geometry of a symmetric (mode I) peel test is shown in Fig. 7. Two identical metal sheets of thickness $h$ are bonded by an adhesive of uniform thickness $t$ and then torn apart by equal and opposite forces per unit width, $P$. When steady-state peeling is achieved, $2 P$ is the macroscopic work per unit of crack extension (per unit width), such that $\Gamma_{\mathrm{ss}}=2 P$. Thus, this test provides a ready method for measuring the steady-state macroscopic toughness for a mode I separation of the joint. The macroscopic toughness is the sum of the energy per unit area consumed in separating the epoxy adhesive plus the plastic dissipation induced in the 




a)

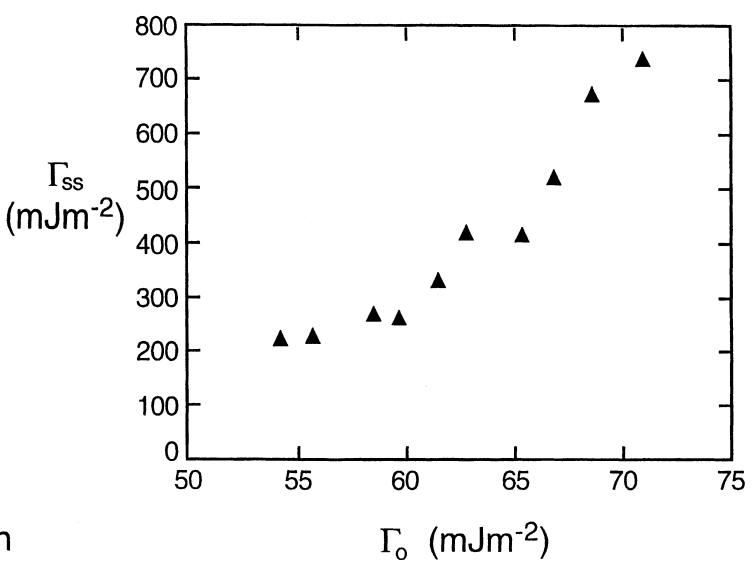

b)

Fig. 8. Measured intrinsic and macroscopic works of fracture for an interface between a self-assembled monolayer and epoxy by Zhuk et al. [13]. (a) Intrinsic interface separation energy, $\Gamma_{0}$, as a function of the adjusted component of the interface chemistry. (b) Experimentally measured macroscopic steadystate interface toughness, $\Gamma_{\mathrm{ss}}$, plotted against intrinsic separation energy.

sheets. A tough joint relies on a strong adhesive to produce accompanying plastic deformation in the sheets.

The EPZ model $[11,12]$ represents the entire thin adhesive layer by the traction-separation relation introduced earlier, characterized by $\Gamma_{0}$ and $\hat{\sigma}$ (cf. Fig. 7). These "interface" parameters must be obtained by fitting model predictions to one set of data obtained for precisely the same thickness of adhesive (since these parameters are expected to depend on the adhesive thickness). The metal sheets are elastic-plastic and represented by a full twodimensional continuum theory. The model enables one to compute the crack growth resistance curve and the steady-state tearing toughness and load, $\Gamma_{\mathrm{ss}}=2 P$. The plasticity enhancement, $\Gamma_{\mathrm{ss}} / \Gamma_{0}$, is a function of the continuum parameters of the sheets and the adhesive parameters, $\Gamma_{0}$ and $\hat{\sigma}$.

A comparison of model prediction and experimental data for two thicknesses of aluminum sheets is presented in Fig. 7 [12]. The adhesive parameters used in the simulation, $\Gamma_{0}=1.4 \mathrm{~kJ} / \mathrm{m}^{2}$ and $\hat{\sigma}=100 \mathrm{MPa}$, were obtained by fitting the model to data on the same type of joint taken under mode I wedge loading. The stress-strain properties of the aluminum sheet material were measured directly and input to the computer simulation. All the parameters of the joint used in carrying out the simulation in Fig. 7 were thus determined independently of the peel test data. The effect of sheet thickness on tearing force is very strong, and the data and model are in reasonable agreement. Very thin sheets undergo less plastic dissipation in bending than thicker sheets primarily because there is less volume of metal to be deformed. For the joints tested (with $h=1$ and $2 \mathrm{~mm}$ ), the leveraging of the adhesive toughness by plastic dissipation in the sheets is more than a factor of ten.

Another illustration of the role of extrinsic plasticity has been provided by a study of crack growth of an interface between a self-assembling monolayer (SAM) and epoxy [13]. The intrinsic separation energy of the SAM-epoxy interface $\Gamma_{0}$ was established by wetting angle measurements. Adjustment of the chemistry of the terminal groups of the SAM was used to systematically change $\Gamma_{0}$ and at the same time to measure the corresponding change in $\Gamma_{\text {ss }}$ with the aid of a macroscopic fracture test. The outcome of the two sets of experimental measurements is given in Fig. 8. The most compelling aspect of the data is the nearly four-fold increase in the macroscopic interface separation energy for less than a $50 \%$ increase in the adhesion energy, $\Gamma_{0}$. It is evident that this large increase cannot be accounted for in the model of Fig. 4 by an increase in $\Gamma_{0}$ without an accompanying increase in $\hat{\sigma}$. The implication is that generally both the adhesion energy and the interface strength play a role in determining the macroscopic interface toughness when extrinsic plasticity occurs.

\section{EPZ MODELS OF DUCTILE FRACTURE}

Ductile fracture is a result of void nucleation, growth and coalescence. Constitutive models of these processes, such as that of Gurson [14], have been developed via specially formulated elements that reproduce the fracture process depicted on the lower right in Fig. 1. The computational models enable simulation of crack initiation and subsequent growth [15-18]. They are effective under both small- and large-scale yielding conditions. These 






(a)



(b)

Fig. 9. A tough pressure vessel steel $(21 / 4 \mathrm{Cr} 1 \mathrm{Mo})$ tested using an edge-cracked specimen in threepoint bending under large-scale yielding $(a / w=0.6)$. (a) Crack growth resistance. (b) Load vs loadpoint displacement. The parameters of the void growth model used to formulate the finite elements were adjusted to give the fit of the model to the data shown [18].

new computational models constitute a comprehensive nonlinear fracture mechanics for mode I crack growth in tough ductile alloys. Much of the motivation driving this development has been the need to assess the integrity of cracked thick sections of piping and pressure vessels in the nuclear power industry. However, the models are applicable to ductile fracture in a wide class of structural problems. Accurate implementation requires that the parameters of the fracture process model (such as the initial void volume fraction and the spacing between the voids) be chosen such that the model reproduces one experimental crack growth history for the alloy under consideration. Once calibrated, the models have proved to be able to reliably predict crack growth under a wide variety of macroscopic geometries and loadings. In particular, in large-scale yielding, the models take into account the very strong role of different geometries in influencing the constraint parallel to the crack front and the accompanying stress triaxiality on crack growth resistance.

One set of results taken from the recent literature (Gao et al. [18]) will be used to illustrate present capabilities. The crack growth resistance as well as load-displacement data are shown in Fig. 9 for a tough pressure vessel steel (2 1/4 Cr $1 \mathrm{Mo}$ ) tested as a single edge-cracked specimen loaded in threepoint bending, as depicted in the inset. The relation of load $P$ to load-point displacement $\Delta$ reflects both the large-scale yielding character of the test and the fact that crack advance takes place. This set of data was used to calibrate the parameters of the void damage model for this material with the result that the initial void volume fraction and void spacing were determined to be $f_{0}=0.0035$ and $D=300 \mu \mathrm{m}$, respectively. With this choice and an accurate representation of the tensile stress-strain data of the undamaged material, the finite element simulation reproduces the load-displacement behavior, including that beyond the peak load, as well as the curve of the $J$-integral vs crack advance, $\Delta a$. Once calibrated, the model has been used to predict the behavior of a surface crack [18] in the wall of a pressure vessel or pipe, which represents one of the primary concerns in assessing fracture integrity. In order to validate the simulations, the specimens shown in Fig. 10(a) were tested under two representative loading histories in which the applied moment and axial force were varied in a prescribed manner. The specimens deformed well into the plastic range thereby falling completely outside the scope of elastic fracture mechanics, as is often the case for components fashioned from tough, ductile alloys. The test was stopped at a prescribed load, the specimen sectioned, and the current crack geometry mapped. Crack advance along the front at two load levels is displayed in Fig. 10(b), along 

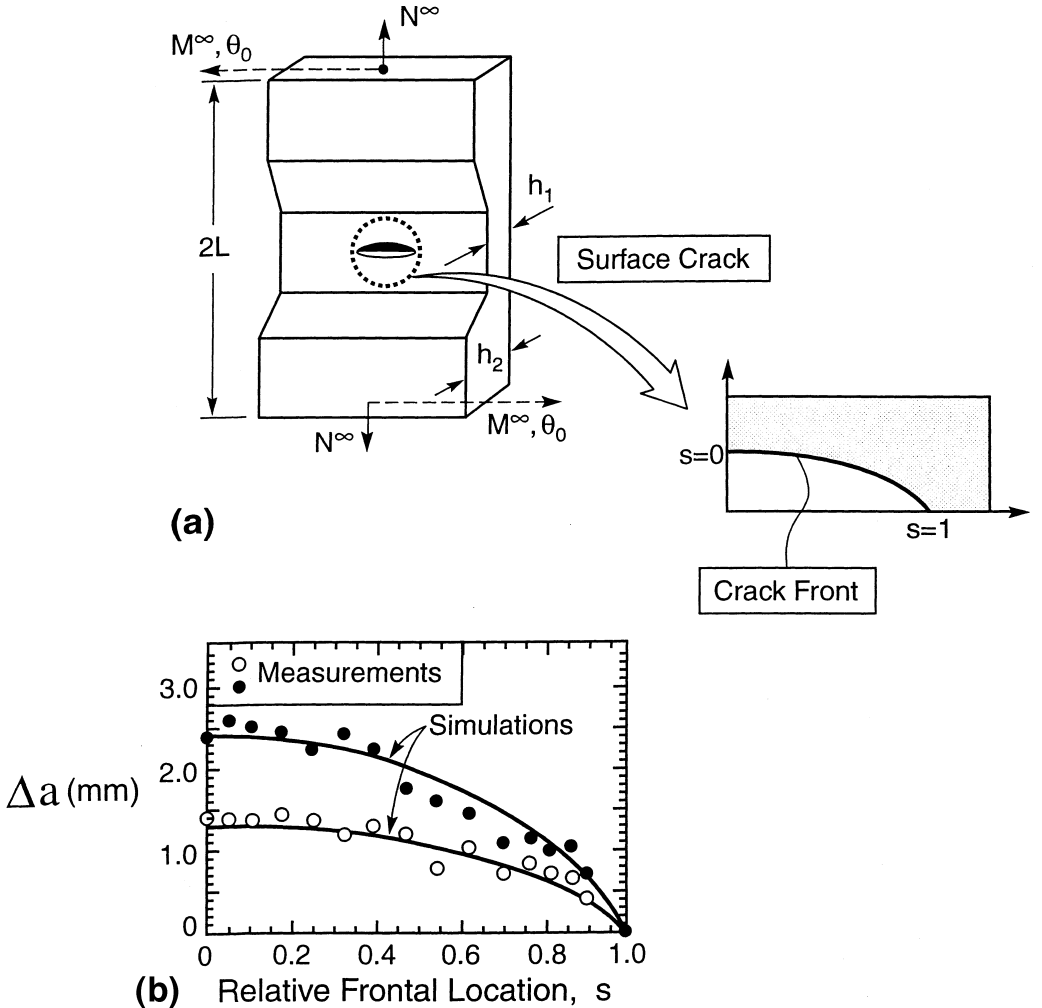

Fig. 10. (a) A specimen employed to investigate the initiation and growth of a surface crack subject to histories of axial load and bending. (b) Crack advance at points along the crack front at two points in a loading history for specimens made from the pressure vessel steel of Fig. 9. Comparison of experimental observation and simulation by Gao et al. [18].

The simulations used the parameters determined by the calibration test in Fig. 9.

with the predictions from the numerical simulations, at the same loads. The three-dimensional aspects of the test are accurately modeled, as is the large effect of the variation of the constraint along the crack front.

This example represents only one of many that validate the utility and accuracy of embedded fracture process models for simulating mode I crack growth in tough, ductile alloys (cf. references cited above). As such, this class of models can be regarded as a qualified tool for the engineering analysis of structural components. A number of important extensions of this approach remain to be implemented. A major challenge, which nevertheless seems achievable, is the extension of the models to encompass fracture from regions of stress concentration, such as blunt notches, as well as from preexisting cracks. In other words, a formulation capable of simulating crack formation, as well as initiation and growth from pre-existing cracks, would comprise a comprehensive framework for the analysis of structural alloys that fracture by void nucleation, growth and coalescence. Already, the existing models have the ability to qualitatively span both crack growth and crack formation, as illustrated by the progressive failure within the neck in a tensile specimen [19]. The present limitation is that the models calibrated using crack growth data are unable to give reliable predictions for crack formation at a blunt notch, and vice versa [20]. The void growth models must be further enhanced and refined before they will be able to cope with the full gamut of conditions under which ductile material failure occurs. Success in extending the models should go a long way to achieving a comprehensive nonlinear fracture mechanics encompassing both crack formation and growth with a greatly enlarged range of applications such as welding failures and metal forming. Another extension would be to very high strength alloys with low strain hardening which are prone to shear localizations and which tend to produce zig-zag crack advance. Coping with these effects would require augmentation of the constitutive model coupled with highly refined meshes near the crack tip to permit crack advance other than in the extended plane of the crack [21]. 

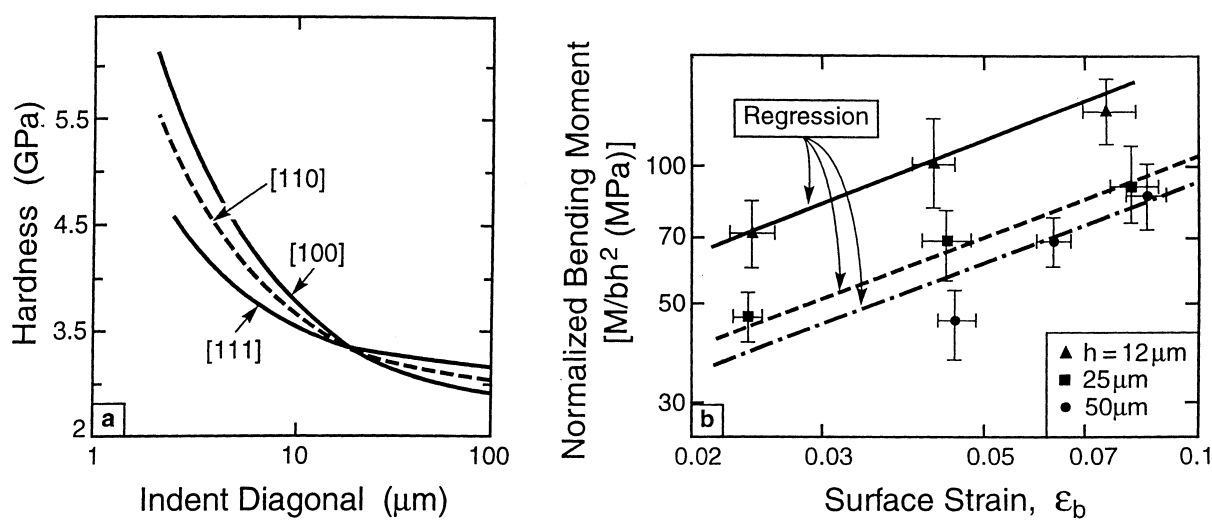

Fig. 11. (a) Hardness (load/indent area) for tungsten single crystals at three orientations as a function of the width of the Vickers indenter (Stelmashenko et al. [24]). (b) Normalized bending moment vs surface strain for three thicknesses $h$ of nickel films tested in pure bending (Stolken and Evans [27]). The indentation data should be independent of the indenter width if there were no material size dependence. Similarly, the normalization used in presenting the bending data is such that the data would be thickness-independent without material size dependence.

\section{ROLE OF SMALL-SCALE PLASTICITY IN STRENGTH AND TOUGHNESS}

A new issue arises in applying the EPZ models to metal films and layers with thicknesses in the micrometer range. Conventional plasticity, which has been devised to describe plastic deformation at large scales, does not incorporate significant sizedependent strengthening effects which arise as the micrometer scale is approached. Recent experimental data on indentation [22-25], wire torsion [26] and film bending [27] have provided evidence of a very strong effect. One set of data for indentation in Fig. 11(a) and another for film bending in Fig. 11(b) are representative. In the absence of any plasticity size effect, the indentation data (as normalized) should be independent of the width of the indent, while the bending data should be independent of the film thickness. Both sets reveal that the effective flow strength of the respective materials is elevated by a factor of between two and three as the scale of deformation is reduced to about $1 \mu \mathrm{m}$. The size-dependent hardening phenomenon is believed to be associated with nonuniform deformation (strain gradients) and the storage of geometrically necessary dislocations [26]. It is self-evident that size-dependent hardening is likely to play an important role in the mechanics of micrometer scale thin films and multilayers involving metals (and possibly polymers too, but that remains to be established). The thin metal layers in the specimens of Fig. 5 range between 0.1 and $4 \mu \mathrm{m}$, and they are subject to highly nonuniform deformation. It seems reasonable to expect that the effects at play in the flow stress elevation in indentation and bending will manifest themselves in the toughness enhancement seen in Figs 5 and 6 . The strong role of yield stress in determining the plasticity contribution to toughness in modeling the specimens has already been emphasized. To some limited extent, the increased flow stress of the thin $\mathrm{Al}-\mathrm{Cu}$ layers may already be taken into account because some estimates of the yield stress were measured directly by indentation of the layer. However, the thickness dependence of flow over the range between 0.1 and $4 \mu \mathrm{m}$ is expected to be considerable and may explain the steepness of the toughness enhancement for the thinnest layers.

We conclude by noting that the approaches discussed in these sections all involve a progression in modeling to finer scales working from the macroscopic down to the microscopic, i.e. the top-down approach. To use the $\mathrm{Al}-\mathrm{Cu}$ multilayer as an example, it is unlikely that dislocation mechanics as presently developed could provide a quantitative model for the $\mathrm{Al}-\mathrm{Cu}$ layers, particularly those as thick as a micrometer. The paper in this issue by Needleman [28] addresses the prodigious increase in the computational complexity in discrete dislocation mechanics modeling as the size of the region increases to a micrometer and beyond. On the other hand, it has been seen that conventional plasticity has its own limitations at this scale, and quantitative modeling almost certainly requires a representation that incorporates plasticity size dependence. The theories of strain gradient plasticity formulated to date [29-32] are themselves phenomenological, although motivated by dislocation theory concepts. The full scope of these theories remains to be explored but there are already studies which indicate important implications for fracture phenomena at the micrometer scale [33].

Acknowledgements-This work was supported in part by NSF Grants CMS-96-34632 and DMR-94-00396 and in part by the Division of Engineering and Applied Sciences, Harvard University. 


\section{REFERENCES}

1. Mohammed, I. and Liechti, K. M., Cohesive zone modeling of crack nucleation at bimaterial corners. Report EMRL 98-20, Research Center for Mechanics of Solids, Structures and Materials, University of Texas at Austin, 1998.

2. Needleman, A., J. appl. Mech., 1987, 54, 525.

3. Tvergaard, V. and Hutchinson, J. W., J. Mech. Phys. Solids, 1992, 40, 1337.

4. Wei, Y. and Hutchinson, J. W., J. Mech. Phys. Solids, 1997, 45, 1137.

5. Evans, A. G., Hutchinson, J. W. and Wei, Y., Acta mater., in press.

6. Dauskardt, R. H., Lane, M. and Ma, Q., Engng Fract. Mech., 1998, 61, 141

7. Wei, Y. and Hutchinson, J. W., Unpublished work on delamination of multilayers.

8. Wei, Y. and Hutchinson, J. W., Int. J. Fract., 1999 95, 1.

9. Suo, Z., Shih, C. F. and Varias, A. G., Acta metall. mater., 1993, 41, 1551.

10. Dauskardt, R. H., Private communication.

11. Yang, Q. D., Thouless, M. D. and Ward, S. M., J Mech. Phys. Solids, 1999, 47, 1337.

12. Yang, Q. D., Thouless, M. D. and Ward, S. M., to be published.

13. Zhuk, A. V., Evans, A. G., Hutchinson, J. W. and Whitesides, G. M., J. Mater. Res., 1998, 13, 3555.

14. Gurson, A. L., J. Engng Mater. Technol., 1977, 99, 2.

15. Rousselier, G., Nucl. Engng Des., 1987, 105, 97.

16. Brocks, W., Kunecke, D. and Sun, D. Z., in Constraint Effects in Fracture: Theory and Applications, ed. M. Kirk and A. Baaker, ASTM STP 1244. ASTM, Philadelphia, PA, 1995.
17. Xia, L. and Shih, C. F., J. Mech. Phys. Solids, 1995, 43, 223.

18. Gao, X., Faleskog, J., Shih, C. F. and Dodds, R. H., Engng Fract. Mech., 1998, 59, 761.

19. Tvergaard, V. and Needleman, A., Acta metall., 1984 32, 157.

20. Pardoen, T. and Delannay, F., Fatigue Fract. Engng Mater. Struct., 1998, 21, 1459.

21. Needleman, A. and Tvergaard, V., J. Mech. Phys. Solids, 1987, 35, 151.

22. Ma, Q. and Clarke, D. R., J. Mater. Res., 1995, 10 853.

23. Nix, W. D. and Gao, H., J. Mech. Phys. Solids, 1998 , 46, 411 .

24. Stelmashenko, N. A., Walls, M. G., Brown, L. M and Milman, Y. V., Acta metall. mater., 1993, 41, 2855.

25. Poole, W. J., Ashby, M. F. and Fleck, N. A., Scripta metall. mater., 1996, 34, 559.

26. Fleck, N. A., Muller, G. M., Ashby, M. F. and Hutchinson, J. W., Acta metall. mater., 1994, 42, 475.

27. Stolken, J. S. and Evans, A. G., Acta mater., 1998, 46, 5109.

28. Needleman, A., Acta mater., 2000, 48, 105.

29. Aifantis, E. C., Int. J. Engng Sci., 1992, 30, 1279.

30. Fleck, N. A. and Hutchinson, J. W., Adv. appl. Mech., 1997, 33, 295.

31. Acharya, A. and Bassani, J. L., in Micromechanics of Plasticity and Damage in Multiphase Materials, IUTAM Symposium, Paris, August 1995.

32. Gao, H., Huang, Y., Nix, W. D. and Hutchinson, J. W., J. Mech. Phys. Solids, 1999, 47, 1239.

33. Wei, Y. and Hutchinson, J. W., J. Mech. Phys. Solids, 1997, 45, 1253 